\title{
ALTERAÇÕES BUCAIS DE IDOSOS INSTITUCIONALIZADOS - REVISÃO DE LITERATURA
}

\section{ORAL PROBLEMS IN ELDERLY PEOPLE IN A NURSING HOME - LITERATURA REVIEW}

\author{
Bárbara Gonçalves de Paula* \\ Maiara Rodrigues Braga de Almeida** \\ Jeane de Fátima Correia Silva Alves***
}

\begin{abstract}
RESUMO
A população brasileira tem aumentado rapidamente e a faixa etária acima dos 60 anos de idade é a que mais cresce. E na medida em que a população envelhece devido a diversos fatores, aumenta a demanda por instituições de longa permanência. O aumento da expectativa de vida dos indivíduos nem sempre se faz acompanhado de saúde, pois, com o processo de envelhecimento, ocorrem alterações funcionais que acarretam maior predisposição ao surgimento de agravos crônicos. Indivíduos idosos, geralmente, consomem maior número de medicamentos, o que pode provocar alterações sistêmicas e também causar efeitos adversos na cavidade bucal. Diante disso, o presente artigo teve como objetivo realizar uma revisão de literatura sobre alterações bucais associadas ao uso de medicamentos em idosos institucionalizados.
\end{abstract}

DESCRITORES: Saúde do idoso institucionalizado • Medicamentos • Doenças da boca.

\section{ABSTRACT}

Brazilian population has increased rapidly lately, specially ages 60 and up and as the population gets older due to one reason or another, the demand for nursing homes increases as well. The increase on life expectancy for elders does not mean any increase in the attention payed on their health. With aging comes some changes that would generate a predisposition to some chronic problems. Elderly people, generally, take a large amount of medication and in some cases its side effects cause some changes in their mouth as well. With that being said, the following article aims to review what has been said about changes occurred in mouths of elderly people who live in nursing homes.

DESCRIPTORS: Health of institutionalized elderly • Medicines • Mouth diseases.

* Acadêmica do Curso de Odontologia do Centro Universitário Newton Paiva/BH-MG, ba-abi@hotmail.com

** Acadêmica do Curso de Odontologia do Centro Universitário Newton Paiva/BH-MG, maiarabraga@hotmail.com

*** Professora Titular I do Curso de Odontologia do Centro Universitário Newton Paiva/BH-MG; Doutora em Patologia Bucal, jeane.alves@newtonpaiva.br 
PAULA BG

ALMEIDA MRB

$A L V E S$ JFCS

ALTERAÇÕES

BUCAIS DE IDOSOS: INSTITUCIONALIZADOS

- REVISÃO DE LITERATURA

$220 \ldots$

REV. ODONTOL. UNIV, CID . SÃO:

PAULO

2014; 26(3): 219-:

26, SET-DEZ

\section{N T RO DUÇÃO}

O rápido envelhecimento da população representou uma importante alteração demográfica do Brasil no final do século XX com aumento acentuado na prevalência da população acima de 60 anos. Pode-se dizer que na primeira década do século XXI a proporção da população idosa no país representava $10 \%$ da população total. Com essa alteração da expectativa de vida da população brasileira surgem novas demandas sociais, sanitárias, econômicas e afetivas. Esse novo contingente populacional apresenta enorme experiência de vida, qualificação e diversas potencialidades para oferecer à sociedade. A população idosa na primeira década deste século atingiu cerca de meio milhão de indivíduos e a expectativa é que, em 2020, esteja em torno de um milhão ${ }^{1}$.

O aumento da expectativa de vida da população nem sempre se faz acompanhado de saúde, pois o processo de envelhecimento acarreta diversas alterações no organismo. Algumas dessas alterações são consideradas patologias que devem ser tratadas com o uso de medicamentos. Geralmente, idosos consomem maior número de medicamentos, o que pode provocar alterações sistêmicas e também causar efeitos adversos na cavidade bucal ${ }^{2}$.

Estudos comprovam que as patologias bucais encontradas em idosos podem ser explicadas pelas diferentes mudanças que envolvem esses pacientes como um todo, sendo elas: o próprio processo de enveIhecimento, mudanças metabólicas, fatores nutricionais, uso de medicamentos, uso de próteses, hábitos psicopatológicos, uso de álcool e uso de tabaco ${ }^{3}$.

Sendo assim, o presente artigo tem como objetivo realizar uma revisão de literatura sobre as alterações bucais, associando-as ao uso de medicações sistêmicas em idosos institucionalizados.

\section{REVISÃO DE LITERATURA}

\subsection{População idosa (acima de 60 anos de idade)}

Estima-se que até 2025 o grupo de idosos no Brasil deverá ter aumentado dez vezes mais. Assim, o Brasil ocupará o sexto lugar quanto ao número de idosos, al- cançando neste mesmo ano, cerca de 32 milhões de pessoas com idade igual ou superior a 60 anos $^{4}$. As estimativas para os próximos 20 anos indicam que a população idosa poderá exceder 30 milhões de pessoas ao final desse período, chegando a representar quase $13 \%$ da população ${ }^{5}$.

O aumento da expectativa de vida da população nem sempre se faz acompanhado de saúde, pois o processo de envelhecimento acarreta mudanças fisiológicas. Embora essas alterações variem de pessoa para pessoa, acarretam maior predisposição ao surgimento de agravos crônicos, tornando muitos desses idosos dependentes no meio social e no meio familiar $^{6}$.

O envelhecimento tem sido tratado como um estado de "terceira idade". O envelhecimento não é uma condição e sim um processo de degradação progressiva e diferencial, abalando todos os seres vivos e o seu curso natural é a morte do organismo. É difícil datar o início do processo pois, de acordo com o grau no qual ele se encontra (biológico, psicológico ou sociológico), a sua gravidade e velocidade variam de pessoa para pessoa. Os indivíduos envelhecem de formas muito diferentes e, assim, podem apresentar diferenças que refletem na idade biológica, na idade social ou na idade psicológica, que é diferente da idade cronológica do indivíduo ${ }^{7}$.

O envelhecimento não é um processo único, não acontece de modo concomitante em todo o organismo e não está associado à existência de uma determinada doença. O avançar da idade biológica envolve vários fatores endógenos e exógenos que devem ser considerados de forma integrada, principalmente, para se chegar a um diagnóstico ${ }^{8}$.

Apesar da velhice não ser sinônimo de doença, sabe-se que o risco do comprometimento da capacidade funcional aumenta, resultando na perda da autonomia e da independência9 ${ }^{9}$.

\subsection{Instituições de longa permanên-} cia

Além do aumento da demanda por serviços de saúde, o envelhecimento populacional também trouxe como consequência o aumento de outras modalidades de 
atendimento voltadas para idosos frágeis ou dependentes, como as instituições de longa permanência para idosos ${ }^{10}$.

No Brasil, percebe-se um aumento significativo das instituições de longa permanência. Estudos revelam que a institucionalização de idosos se dá por vários fatores, como: solidão associada à viuvez, menor oportunidade de emprego, menor suporte social e baixa renda e esses fatores se tomaram mais evidentes nos últimos $\operatorname{anos}^{11}$

Estima-se que 113 mil idosos eram residentes de domicílios coletivos. Desse total, 107 mil idosos eram residentes em instituições de longa permanência e, segundo o Ministério de Desenvolvimento Social e Combate à Fome, foram financiadas pela União, em 2005, 1.146 instituições para 24.859 idosos $^{12}$.

O termo asilo tem sido definido como casa de assistência social onde são recoIhidas, para sustento ou também para educação, pessoas pobres e desamparadas, como mendigos, crianças abandonadas, órfãos e velhos. Outros termos têm sido usados para denominar locais de assistência a idosos, como clínica geriátrica, abrigo, lar e casa de repouso. Recentemente, foi proposta a denominação de instituições de longa permanência para idosos (ILPI), que define o estabelecimento para atendimento integral a idosos ${ }^{13}$.

O Estatuto do Idoso define que "a assistência integral na modalidade de entidade de longa permanência será prestada quando verificada inexistência de grupo familiar, casa-lar, abandono ou carência de recursos financeiros próprios ou da família" e que "as instituições que abrigarem idosos são obrigadas a manter padrões de habitação compatíveis com as necessidades deles, bem como provê-los com alimentação regular e higiene indispensável às normas sanitárias e com estas condizentes, sob as penas da lei ${ }^{14 \prime}$. -

A Política Nacional do Idoso define que o atendimento ao idoso deve ser realizado, primeiramente, pela sua própria família, e destaca que o serviço prestado pelas ILPI pode ser considerado como uma alternativa assistencial para muitos idosos ${ }^{15}$ visto que as famílias têm passado por transformações na constituição e no número de filhos. Essas mudanças têm afetado as funções familiares de cuidar e amparar os idosos, principalmente aqueles que não são mais capazes de realizar as atividades básicas da vida diária sozinhos $^{16}$.

No Brasil não há normas específicas para o cuidado da saúde bucal de idosos institucionalizados. Não se sabe ao certo se existe diferença na condição bucal dos idosos não institucionalizados e dos institucionalizados. Sabe-se que há uma deficiência no conhecimento referente ao cuidado da saúde bucal dos mesmos ${ }^{17}$.

\subsection{Alterações bucais relacionadas ao uso de medicamentos}

Devido aos problemas crônicos de saúde, os idosos utilizam com frequência os serviços de saúde e consomem um grande número de medicamentos ${ }^{18}$. Estudos revelam que grande número de idosos consomem pelo menos um tipo de medicamento e muitos deles fazem uso de cinco ou mais associações, o que pode trazer consequências e/ou benefício para a saúde ${ }^{19}$.

O uso contínuo de medicamentos produz as mais distintas reações no organismo, sistêmicas e locais. Além disso, essas reações podem causar efeitos adversos na cavidade bucal, potencializando o desenvolvimento de lesões bucais. Essas lesões bucais, associadas ao uso de medicamentos, podem estar relacionadas a mecanismos imunológicos ou a mecanismos não imunológicos ${ }^{20}$.

Os idosos constituem o maior grupo de consumidores de medicamentos per capita do mundo. Os medicamentos cardiovasculares, analgésicos, sedativos e tranquilizantes são os mais consumidos pela população idosa ${ }^{21}$.

Tem sido descrito que o processo de envelhecimento pode influenciar a prevalência de doenças bucais. Com o avançar da idade a mucosa bucal, geralmente, torna-se mais susceptível a danos mecânicos. Além disso, algumas doenças sistêmicas e o próprio uso de medicamentos podem causar efeitos negativos na secreção salivar, resultando assim em um aumento do risco para o aparecimento de lesões bucais na população idosa. Como consequência, os pacientes idosos necessitam de um acompanhamento odontológico para
PAULA BG

ALMEIDA MRB

ALVES JFCS

ALTERAÇÕES

BUCAIS DE IDOSOS

INSTITUCIONALIZADOS

- REVISÃO DE

LITERATURA

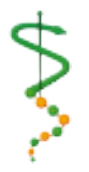

REV, ODONTOL.

UNIV, CID, SÃO

PAULO

$2014 ; 26(3): 219-$

26, SET-DEZ 
PAULA BG

ALMEIDA MRB

$A L V E S$ JFCS

ALTERAÇÕES

BUCAIS DE IDOSOS INSTITUCIONALIZADOS

- REVISÃO DE LITERATURA

$222 \ldots$

REV, ODONTOL. UNIV, CID, SÃO

PAULO

$2014 ; 26(3): 219-$

26, SET-DEZ avaliar, prevenir e tratar essas alterações bucais associadas ao uso de medicamentos sistêmicos ${ }^{22,23,24}$. Ainda que haja a tendência em tratar a cavidade bucal como um ponto de referência autônoma do resto do corpo, a saúde bucal é uma parte integrante da saúde geral do indivíduo ${ }^{25}$.

A cavidade bucal sofre muitas alterações durante o envelhecimento, como a retração dos tecidos periodontais por redução da celularidade, diminuição da espessura da mucosa e modificações na superfície da língua ${ }^{26}$.

Dentre as lesões associadas ao uso de medicamentos que afetam pacientes idosos podemos destacar: Candidose, Xerostomia e Hiperplasia gengival.

\subsubsection{Candidose}

É uma infecção fúngica mais comum na cavidade bucal, sendo que a espécie mais encontrada é a Candida albicans ${ }^{27}$ que é um fungo dimórfico, ou seja, pode se apresentar na forma de levedura ou hifa, sendo a última a patogênica ${ }^{28}$. Clinicamente, a lesão pode se apresentar como uma membrana branca, amarelada ou acinzentada, de superfície irregular e briIhante, que pode ser facilmente removida à raspagem. A lesão pode recobrir áreas extensas em forma de placa ou ainda pequenas áreas puntiformes em regiões isoladas $^{29}$.

Uma das lesões mais comum na população idosa é a candidose, devido ao ambiente favorável para a colonização e proliferação de Candida spp. Os medicamentos capazes de desencadear o surgimento dessa lesão são os imunossupressores, os antibióticos de amplo espectro, corticoides (beclometasona e triacinolona) e omeprazol ${ }^{30}$

A Candidose Pseudomembranosa aguda é uma das variantes clínicas mais comuns de manifestação da doença, podendo acometer pessoas em qualquer idade, mas principalmente os indivíduos debilitados ou portadores de doenças crônicas $^{31}$. Apresenta-se, clinicamente, como placas ou nódulos branco-amarelados ${ }^{32}$ que são facilmente removidas com gazes ou cotonete co $^{33}$.

A Candidose Eritematosa é uma lesão sintomática, devido às numerosas erosões propagadas pela mucosa e à inflamação presente, causando uma sensibilidade intensa. A localização preferencial dessa variante clínica ocorre ao longo do dorso da língua. Geralmente, as lesões são assintomáticas, causando apenas ardência na ingestão de alimentos ácidos ou quentes ${ }^{34}$. Apresenta algumas variantes clínicas que acometem, frequentemente, pacientes idosos, como Atrófica Crônica e Queilite Angular ${ }^{35}$.

A Candidose Atrófica Crônica consiste em alterações inflamatórias encontradas, comumente, debaixo da prótese total maxilar $^{36}$ Esse tipo de lesão pode acometer até $65 \%$ dos idosos que utilizam prótese, principalmente a prótese total superior ${ }^{37}$ e pode aparecer em menor frequência na mucosa de suporte da prótese parcial removível ${ }^{38}$. Os fatores etiológicos responsáveis pelo aparecimento da Candidose Eritematosa Atrófica Crônica são vários, entre eles o traumatismo crônico, próteses mal adaptadas, a não remoção da prótese durante a noite e a má higiene do pacien$\mathrm{te}^{33}$.

A avulsão de elementos dentários e/ou abrasão dos elementos remanescentes podem ocasionar a diminuição da dimensão vertical, o que pode predispor à ocorrência de queilite angular ${ }^{39}$. Em idosos que apresentam perda de dimensão vertical de oclusão é comum o acúmulo de saliva nas comissuras labiais, o que predispõe à proliferação de Candida spp, ocasionando a ocorrência da lesão ${ }^{40}$. Alguns medicamentos como atrovastatina, busulfan, clofazimina, metildopa, ritonavir, sais de ouro, sinvastatina, tetraciclina, indinavir e vitamina $A$, têm sido associados à ocorrência de queilite angular. Esses medicamentos propiciam o acúmulo de saliva na comissura labial, bem como potencializam o crescimento de microorganismos principalmente a Candida albicans na cavidade bucal, além de diminuir a resposta imunológica, favorecendo, assim, o desenvolvimento da Queilite Angular ${ }^{35}$.

\subsubsection{Xerostomia}

A função adequada das glândulas salivares é essencial para todos os aspectos das funções bucais. A saliva tem papel importante na proteção dos tecidos bucais, lubrificando a mucosa, prevenindo a desmineralização e promovendo a remi- 
neralização dos dentes. A redução do funcionamento das glândulas salivares pode propiciar o desenvolvimento de xerostomia. O principal sintoma dessa alteração é a sensação de boca seca. Além disso, os pacientes podem apresentar redução na produção da amilase salivar, o que prejudica a digestão de carboidratos na cavidade bucal e, consequentemente, dificulta a deglutição e posterior digestão do bolo alimentar $^{35,40}$. Com o envelhecimento, as glândulas salivares podem sofrer alterações degenerativas, o que também propicia diminuição da quantidade e da viscosidade da saliva secretada, especialmente em repouso ${ }^{30,41}$.

Estudos têm demonstrado que a xerostomia, presente em grupos de idosos, pode estar relacionada ao uso de anti-hipertensivos e digitálicos ${ }^{42,43}$. Outros medicamentos associados à redução do fluxo são os analgésicos, anticonvulsivantes, anticolinérgicos, antieméticos, anti-histamínicos, descongestionantes, diuréticos e psicotrópicos ${ }^{35}$.

A diminuição do teor hídrico, fibrose e perda da elasticidade da mucosa pode induzir o desenvolvimento de erosões e úlceras na mucosa oral, que é, frequentemente, causa de grande desconforto entre os idosos ${ }^{40}$.

\subsubsection{Hiperplasia Gengival Medi- camentosa}

A Hiperplasia Gengival Medicamentosa refere-se ao aumento gengival induzido por drogas. É caracterizada pelo aumento do volume gengival causado pelo uso de medicamentos que interferem com o metabolismo dos fibroblastos e do colágeno. Diversos medicamentos, incluindo o ácido tranexâmico, anlodipina, cetoconazol, ciclosporina, cotrimoxazol, eritromicina, felodipina, fenitoína, nicardipina, verapamil, vigabratina, nifedipina e pirimidona têm sido associados ao sobrecrescimento gengival ${ }^{44,45,46}$.

A ocorrência de hiperplasia gengival induzida por medicamentos em pacientes desdentados não é comum e, quando ocorre, está relacionada a fatores irritantes locais como próteses e a deposição de placa bacteriana sobre elas ${ }^{47}$.

A hiperplasia gengival induzida por drogas geralmente se inicia entre o primeiro e o terceiro mês da terapia com o medicamento, aumentando somente durante os próximos doze a dezoito meses ${ }^{48}$.

\section{CONSIDERAÇÕES FINAIS}

A ampliação dos conhecimentos sobre lesões bucais associadas ao uso de medicamentos poderá contribuir para melhorar o atendimento odontológico aos pacientes idosos, principalmente, daqueles que vivem em ILPI sem a assistência familiar.

Diante dos fatos, evidencia-se a importância de garantir aos idosos institucionalizados uma boa qualidade de vida, uma vez que esta já é amplamente comprometida em função do menor suporte social.

O conhecimento sobre a realidade das populações idosas no Brasil torna-se importante para que o cirurgião-dentista possa ser capaz de planejar medidas para manutenção da saúde bucal desses pacientes, em razão das alterações que podem ocorrer na cavidade bucal, surgindo os medicamentos sistêmicos de uso prolongado como fator predisponente. O cirurgião-dentista tem de ser capaz de reconhecer essas alterações e associá-las ao uso de medicamentos para delinear programas de intervenção à saúde que beneficiam esse grupo populacional.
PAULA BG

ALMEIDA MRB

ALVES JFCS

ALTERAÇÕES

BUCAIS DE IDOSOS

INSTITUCIONALIZADOS

- REVISÃo DE

LITERATURA

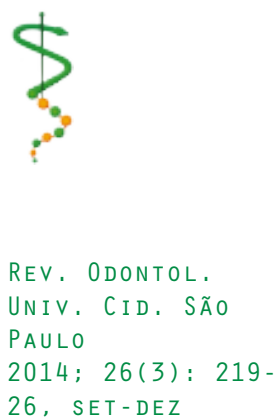


PAULA BG

ALMEIDA MRB

ALVES JFCS

ALTERAÇÕES

BUCAIS DE IDOSOS INSTITUCIONALIZADOS

- REVISÃO DE LITERATURA
1. Lebrão ML. SABE - Saúde, bem-estar e envelhecimento: o projeto Sabe no município de São Paulo: uma abordagem inicial. Brasília: Organização Pan-Americana da Saúde; 2003.

2. Ribeiro DG, Silva MM, Arioli FiIho JN, Nogueira SS. A saúde bucal na terceira idade Salusvita 2009 28(1):101-11.

3. Chaimowicz F. A saúde dos idosos brasileiros às vésperas do século XXI: problemas, projeções e alternativas. Rev Saúde Pública 1997 abr;31(2):184-200.

4. Fantasía J. Diagnóstico y tratamiento de las lesiones orales más comunes encontradas en ancianos. Toma de decisiones clínicas en odontología geriátrica: clínicas odontológicas de norteamérica: McGraw-Hill Interamericana; 1997.

5. Andrews GA. Los desafíos del proceso de envejecimiento en las sociedades de hoy y del futuro. Encuentro Latinoamericano y Caribeño sobre las Personas de Edad; Santiago: Celade; 1999. p. 274-56.

6. Brasil. Ministério da Saúde. Portaria $\mathrm{n}^{\circ} 1.395$ de 10 de dezembro de 1999. Institui a Política Nacional de Saúde do Idoso. Brasília, Diário Oficial da União; 1999.

7. Torres GV, Reis LA, Reis LA, Fernandes $M H$, Xavier TT. Relação entre funcionalidade familiar e capacidade funcional de Idosos dependentes no município de Jequié (BA). Rev baiana saúde pública 2010 jan-mar;34(1):

8. Castro IR. Psicologia do desenvolvimento: envelhecimento. 2014 [Acesso em 28 de maio de 2014]; Disponível em: http://metamorfosepsicdesenvolvimento.wordpress.com.
9. Palácios J. Mudança e desenvolvimento durante a idade adulta e a velhice. In: Coll C, Palacios J, Marchesi A. Desenvolvimento psicológico e educação, psicologia evolutiva. 2 ed. Porto Alegre: Artmed; 2004.

10. Queiroz ZPV. Cuidando do idoso: uma abordagem social. Mundo saúde (Impr) 200 jul.-ago.;24(4):246-8.

11. Silva AE, Menezes EAG, Coelho TOA, Moraes EN, editors. Aspectos bio-psico-sociais dos idosos institucionalizados na casa do ancião da cidade ozanan, no ano de 2005, em Belo Horizonte. $8^{\circ}$ Encontro de Extensão da UFMG; 200503 a 08 de out de 2005; Belo Horizonte: Universidade Federal de Minas Gerais.

12. Camarano AA. Idosos brasileiros: indicadores de condições de vida e de acompanhamento de políticas. Brasília: Presidência da República; 2005.

13. Sociedade Brasileira de Geriatria e Gerontologia. Seção São Paulo. Instituição de longa permanência para idosos: manual de funcionamento. São Paulo: SBGG; 2004.

14. Brasil. Ministério da Saúde. Estatuto do idoso Brasília: Ministério da Saúde; 2003.

15. Leake JL. An index of chewing ability. J Public Health Dent 1990 Summer;50(4):262-7.

16. Souza RF, Patrocinio L, Pero AC, Marra J, Compagnoni MA. Reliability and validation of a Brazilian version of the Oral Health Impact Profile for assessing edentulous subjects. J Oral Rehabil 2007 Nov;34(11):821-6.

17. Mello ALSF. Promovendo o cuidado à saúde bucal do idoso: revelando contradições no processo de cuidar e incorporando melhores práticas a partir do contexto da instituição de longa permanência para idosos [Dissertação]. Florianópolis: Universidade Federal de Santa Catarina; 2005. 
18. Lima CMF. Epidemiologia do enveIhecimento no Brasil. In: IRouquayrol MZ, Almeida Filho N. 6. ed. Rio de Janeiro: Medsi; 2003. p. 499-513.

19. Castellar JI, Karnikowski MGO, Vianna LG, Nóbrega OT. Estudos da farmacoterapia prescrita a idosos em instituições brasileiras de longa permanência. Acta Med Port 2007 20(1):97-105.

20. Abdollahi M, Radfar M. A review of drug-induced oral reactions. J Contemp Dent Pract 2003 Feb 15;4(1):1031.

21. Corgel JA. Periodontal treatment of geriatric patients. In: Carranza J, Newman MG. Clinical periodontology. 8. ed. Philadelphia: Saunders; 1996. p. 423-6.

22. Rezende A, Bispo MCR, Oliveira AC. Saúde bucal na terceira idade: egressos do curso de Odontologia frente às mudanças nos modelos de atuação pública e privada. Rev Reg Araçatuba Assoc Paul Cir Dent 2001 jul.-dez. ;22(2):1-6.

23. Avcu N, Ozbek M, Kurtoglu D, Kurtoglu E, Kansu O, Kansu H. Oral findings and health status among hospitalized patients with physical disabilities, aged 60 or above. Arch Gerontol Geriatr 2005 Jul-Aug;41(1):69-79.

24. Veloso KMM, Costa LJ. Avaliação clínica e orientação terapêutica das manifestações fisiológicas e patológicas da cavidade bucal de pacientes idosos de São Luís do Maranhão. 2003 [Acesso em: 15 de abril de 2006. Disponível em: http://www.ripsa.org.br/ en/lis/resource/17668.

25. Cunningham SJ, Hunt NP. Quality of life and its importance in orthodontics. J Orthod 2001 Jun;28(2):152-8.

26. Silva SO, Trentin MS, Linden MSS, Carli JP, Silveira Neto N, Luft LR. Saúde bucal do idoso institucionalizado em dois asilos de Passo Fundo - RS. RGO (Porto Alegre) 2008 jul-set;56(3):303-8.
27. Williams DW, Potts AJ, Wilson MJ, Matthews JB, Lewis MA. Characterisation of the inflammatory cell infiltrate in chronic hyperplastic candidosis of the oral mucosa. J Oral Pathol Med 1997 Feb;26(2):83-9.

28. Bunetel L, Bonnaure-Mallet M. Oral pathoses caused by Candida albicans during chemotherapy: update on development mechanisms. Oral Surg Oral Med Oral Pathol Oral Radiol Endod 1996 Aug;82(2):161-5.

29. Boraks S. Diagnóstico bucal. 3. ed. São Paulo: Artes Médicas; 2001.

30. Smith RG, Burtner AP. Oral side-effects of the most frequently prescribed drugs. Spec Care Dentist 1994 May-Jun;14(3):96-102.

31. Shafer WG, Hine MK, Levy BM. Tratado de patologia bucal. 4. ed. Rio de Janeiro: Guanabara Koogan; 1987.

32. Araújo NS, Araújo VC. Patologia bucal. São Paulo: Artes médicas; 1994.

33. Regezi JA, Sciubba JJ. Patologia bucal- correlações clinicopatológicas. 3. ed. Rio de Janeiro: Guanabara Kooga; 2000.

34. Martins Neto M, Danesi CC, Unfer DT. Candidíase bucal: revisão de literatura. Rev Cent Ciências da Saúde 200531 (1 e 2):16-26.

35. Neville BW, Damm DD, Allen CM. Patologia oral e maxilofacial. 2. ed. Rio de Janeiro: Guanabara Koogan; 2004.

36. Pindborg JJ. Atlas das doenças da mucosa oral. 3. ed. São Paulo: Editorial Médica Panamericana; 1981.

37. Sonis St, Fazio Rc, Fang L. Princípios e prática de medicina oral. 2. ed. Rio de Janeiro: Guanabara Koogan; 1996.

38. Scully C. Atlas de diagnóstico bucal. São Paulo: Santos; 1992.

39. Boraks S. Distúrbios bucais na terceira idade. In: Brunetti R, Montenegro FLB. Odontogeriatria: noções de interesse clínico. São Paulo: Artes Médicas; 2002.
PAULA BG

ALMEIDA MRB

ALVES JFCS

ALTERAÇÕES

BUCAIS DE IDOSOS

INSTITUCIONALIZADOS

- REVISÃO DE

LITERATURA
REV. ODONTOL.

UnIV. CID. São

PAULO

2014; 26(3): 219 -

26, SET-DEZ 
PAULA BG ALMEIDA MRB

$A L V E S$ JFCS

ALTERAÇÕES BUCAIS DE IDOSOS INSTITUCIONALIZADOS

- REVISÃO DE LITERATURA
40. Nevalainen MJ, Narhi TO, Ainamo A. Oral mucosal lesions and oral hygiene habits in the home-living elderly. J Oral Rehabil 1997 May;24(5):332-7.

41. Pucca Junior, Alfredo G. Saúde bucal e reabilitação na terceira idade. Odontol Mod 1995 out.-dez. ;22(4):27-8.

42. Pajukoski H, Meurman JH, Snellman-Grohn S, Keinanen S, Sulkava R. Salivary flow and composition in elderly patients referred to an acute care geriatric ward. Oral Surg Oral Med Oral Pathol Oral Radiol Endod 1997 Sep;84(3):265-71.

43. Kikutani T. Investigation of administred drugs and intra oral side effect in elderly dental patients. Nippon Dent Univ Ann Public 1995 30(1):70.

44. Loureiro CCS, Adde CA, Perez FEG, Penha SS. Efeitos adversos de medicamentos tópicos e sistêmicos na mucosa bucal. Rev Bras Otorrinolaringol 2004 jan.;70(1):106-11.
45. Marcucci G. Fundamentos de odontologia: estomatologia Rio de Janeiro: Guanabara Koogan; 2005.

46. Marshall RI, Bartold PM. A clinical review of drug-induced gingival overgrowths. Aust Dent J1999 Dec;44(4):21932.

47. McCord JF, Sloan P, Quayle AA, Hussey DJ. Phenytoin hyperplasia occurring under complete dentures: a clinical report. J Prosthet Dent 1992 Oct;68(4):569-72.

48. Vieira MLSO, Calvassan KH, Martins Júnior W, Grisi MFM, Salvador SL. Patogenia de hiperplasia gengival medicamentosa: revisão da literatura J Bras Endo/Perio 2001 jan/mar;2(4):15-22.

Recebido em 02/12/2014

Aceito em 11/03/2015 